\title{
Reciprocal Relation between Special Relativity and Quantum Mechanics and Correspondence between Continuous and Discrete Motion
}

\author{
Mushfiq Ahmad
}

\begin{abstract}
We have observed a reciprocal relation between Planck's hypothesis and Einstein's postulate. Particle velocity and de Broglie wave velocity are also reciprocally related. We have postulated reciprocal symmetry and shown that it relates Special Relativity to quantum mechanics. Reciprocal symmetry implies that there is a boundary value velocity c, which is the limiting value and which is also constant under addition. This corresponds to Einstein's postulate. We have, then, developed reciprocal symmetric law of addition of velocities. In case of collinear velocities it agrees with LorentzEinstein law of addition. We have found the expression for the distance traveled. It agrees with the hyperbolic transform of the distance in Galilean space. If velocities are replaced by reciprocal velocities the distance traveled still gives the correct value, but only for integral multiples of unit time. For non integral values, the distance is complex.
\end{abstract}

Index Terms-Reciprocal symmetry, reciprocal symmetric addition, reciprocal symmetric multiplication, Lorentz transformation

\section{INTRODUCTION}

In 1900 Planck's hypothesis set a lower limit to energy quantum. In 1905 Einstein suggested velocity has an upper limit. If a quantity has an upper limit, its reciprocal has a lower limit. This suggests a reciprocal relation between Planck's hypothesis and Einstein's postulate.

If a particle travels with velocity $v$, the corresponding matter de Broglie wave travels with the reciprocal velocity $v^{*}=c^{2} / v$. We intend to study the implications of this reciprocity in this paper. We shall see that the reciprocal relation gives a correspondence between discrete and continuous quantities.

\section{RECIPROCAL SYMMETRY}

To reflect the symmetry mentioned above, we postulate that the law of addition (composition) of velocities $\oplus$ should fulfil the symmetry condition

Postulate:

$$
u \oplus v=(u *) \oplus(v *)
$$

(1) implies[1]

$$
c \oplus v=c
$$

Manuscript received May 9, 2012; revised June 12,2012

M. Ahmad is with the Department of Physics, Rajshahi University, Rajshahi-6205, Bangladesh (e-mail: mushfiqahmad@gmail.com).

\section{MOTION IN ONE DIMENSION}

Two definitions of $\oplus(\oplus$ and $\oplus *)$ fulfil requirement (1)

$$
u \oplus v=(u *) \oplus\left(v^{*}\right)=\frac{u+v}{1+u \cdot v / c^{2}}
$$

and

$$
u \oplus * v=(u *) \oplus *(v *)=\frac{u v+c^{2}}{u+v}
$$

\section{HyPERBOLIC TRANSFORMATION AND RELATIVISTIC VELOCITY}

We relate Galilean velocity $U$ to the corresponding relativistic [2] velocity u by[3]

$$
U / c=\ln \frac{1+u / c}{\sqrt{1-(u / c)^{2}}} \text { where } u / c=\tanh (U / c)
$$

The Galilean relative velocity $W=U-V$ becomes, using (5)

$$
W=U-V=c \cdot \ln \frac{1+u / c}{\sqrt{1-(u / c)^{2}}} \frac{1-v / c}{\sqrt{1-(v / c)^{2}}}=c \cdot \ln \frac{1+w / c}{\sqrt{1-(w / c)^{2}}}
$$

where the corresponding relativistic sum is[4]

$$
w=u \oplus(-v)=\frac{u-v}{1-u \cdot v / c^{2}}
$$

\section{Distance And Velocity In HyPERbolic SPACE}

Corresponding to (5) we can write for $\mathrm{X} / \mathrm{L}$

$$
X / L=\ln \psi(x / L)=\ln \frac{1+x / L}{\sqrt{1-(x / L)^{2}}}
$$

where $x / L=\tanh (X / L)(5)$

If $V t=X$ and $L=c T$, where [5] $\mathrm{T}$ is a constant unit 
time, we have

$(V / c) .(t / T)=X / L=\ln \{\psi(v / c)\}^{t / T}=\ln \psi((t / T) \otimes(v / c))$ $=\ln \psi(x / L)(30)$

where [6]

$$
x / L=(t / T) \otimes(v / c)=\frac{\left(\frac{1+v / c}{1-v / c}\right)^{t / T}-1}{\left(\frac{1+v / c}{1-v / c}\right)^{t / T}+1}
$$

Using (8) we have

$$
x=c T\{(t / T) \otimes(v / c)\}
$$

In the limit $c \rightarrow \infty$, (9) gives

$$
x \underset{c \rightarrow \infty}{\longrightarrow} c T\{(t / T) .(v / c)\}=v t \underset{c \rightarrow \infty}{\longrightarrow} V t
$$

\section{RECIPROCAL VELOCITY AND DISCRETE TIME}

We exploit the symmetry under reciprocal inversion $v / c \rightarrow v * / c=c / v$ in (8) to define ${ }^{[1]}$ distance $\tilde{x}$ in place of $x$

$$
\begin{aligned}
\tilde{x} / L=(t / T) \otimes(v * / c) & =\frac{\left(\frac{1+c / v}{1-c / v}\right)^{t / T}-1}{\left(\frac{1+c / v}{1-c / v}\right)^{t / T}+1} \\
& =\frac{(-1)^{t / T}\left(\frac{1+v / c}{1-v / c}\right)^{t / T}-1}{(-1)^{t / T}\left(\frac{1+v / c}{1-v / c}\right)^{t / T}+1}
\end{aligned}
$$

Therefore, from (11), for $t / T=2 . n$ where $n$ is an integer and when $c \rightarrow \infty$

$$
\begin{aligned}
x & =\tilde{x}=L .(2 \cdot n) \otimes\left(v^{*} / c\right) \underset{c \rightarrow \infty}{\longrightarrow} L \cdot(2 \cdot n) \cdot(v / c) \\
& =(2 . n) . v T \underset{c \rightarrow \infty}{\longrightarrow} 2 . n . V . T
\end{aligned}
$$

\section{RECIPROCAL AlgEBRA AND DISCRETE TIME}

We have [7] two laws of addition (3) and (4) which have reciprocal symmetry. Corresponding to (4) we can define distance corresponding to (11)

$$
\tilde{z} / L=(t / T) \otimes *(v / c)=\frac{\left(\frac{v / c+1}{v / c-1}\right)^{t / T}+1}{\left(\frac{v / c+1}{v / c-1}\right)^{t / T}-1}
$$

$$
=\frac{(-1)^{t / T}\left(\frac{1+v / c}{1-v / c}\right)^{t / T}+1}{(-1)^{t / T}\left(\frac{1+v / c}{1-v / c}\right)^{t / T}-1}
$$

When $t=(1 / 2+n) \cdot 2 \cdot T$ where $n$ is an integer, we have

$$
\tilde{z} / L=x / L=(2 . n+1) \otimes *(v / c)
$$

$$
=\frac{\left(\frac{1+v / c}{1-v / c}\right)^{2 . n+1}-1}{\left(\frac{1+v / c}{1-v / c}\right)^{2 . n+1}+1}
$$

Corresponding to (12), we have

$$
\begin{aligned}
\tilde{z} & =x \underset{c \rightarrow \infty}{\longrightarrow} L \cdot(2 \cdot n+1) \cdot(v / c) \\
& =(1 / 2+n) \cdot(2 \cdot T \cdot v)
\end{aligned}
$$

\section{CONCLUSION}

We have shown a reciprocal relation between relativity and quantum mechanics. We have then defined reciprocal symmetry and developed reciprocal symmetric kinematics. By replacing velocity by its reciprocal we have been able to find the distance covered by a moving body in terms of reciprocal velocity. It agrees with normal real distance when the time of travel is an even multiple of unit time. Using the reciprocal definition of multiplication, we again get the correct value of distance, but only for odd integral multiples of unit time. For non integral values of time the distance travelled is complex.

\section{REFERENCES}

[1] M. Ahmad and M. O. G, Talukder. Phys. Essays vol. 24, no. 4, pp. 593-597, 2011.

[2] M. Ahmad, Physics Essays, vol. 22, no. 1, 2009.

[3] M. Ahmad, arXiv:math-ph/0612019v1

[4] M. Ahmad and M. S. Alam, Phys. Essays, vol. 22, pp. 164-167, 2009.

[5] M. Ahmad, Journal of Sc. Res, vol. 1, no. 2, pp. 270-274.

[6] M. Ahmad, Rajshahi University J. of Sci. vol. 38, pp. 01-08, 2012.

[7] M. Ahmad and M. O. G. Talukder, Rajshahi University J. of Sci., vol. 38, pp. 09-12, 2012. 